\title{
Molecular Characterization of CTX-M Producing Salmonella Isolates with Concurrent Resistance to Ciprofloxacin and Cefotaxime from Slaughtered Chicken Carcasses in Qingdao, China
}

Wei Wang ${ }^{1}$, Jin Xu ${ }^{1}$, Li Zhao ${ }^{2}$, Yujie Hu${ }^{1}$, Yao Bai ${ }^{1}$, Xin Gan ${ }^{1}$, Yinping Dong ${ }^{1}$, Zixin Peng ${ }^{1}$, Shaofei Yan ${ }^{1}$, Menghan Li ${ }^{1}$, Fengqin Li ${ }^{1 * \#}$ and Séamus Fanning ${ }^{1,3,4^{*} \sharp}$ ${ }^{1}$ NHC Key Laboratory of Food Safety Risk Assessment, China National Center for Food Safety Risk Assessment, Beijing, China

${ }^{2}$ School of Public Health, Shandong University, Jinan, China

${ }^{3}$ UCD-Centre for Food Safety, School of Public Health, Physiotherapy and Sports Science, University College Dublin, Belfield, Dublin D04 N2E5, Ireland

${ }^{4}$ Institute for Global Food Security, School of Biological Sciences, Queen's University Belfast, Stranmillis Road, Belfast BT9 5AG, Northern Ireland

*Corresponding authors: Fengqin Li, NHC Key Laboratory of Food Safety Risk Assessment, China National Center for Food Safety Risk Assessment, Beijing, China, Tel: (+86)01067776356; E-mail: lifengqin0224@163.com

Séamus Fanning, UCD-Centre for Food Science, University College Dublin, Belfield, Ireland, Tel: (+353-1) 716 2869; E-mail: sfanning@ucd.ie

Received date: January 29, 2019; Accepted date: April 2, 2019; $\quad$ Published date: April 9, 2019

Copyright: (2019 Wang W, et al. This is an open-access article distributed under the terms of the Creative Commons Attribution License, which permits unrestricted use, distribution, and reproduction in any medium, provided the original author and source are credited. \#Both authors contributed equally.

\section{Abstract}

Objectives: To describe the molecular characterization of CTX-M ESBL (Extended-spectrum Beta-lactamase)producing Salmonella co-resistant ciprofloxacin and cefotaxime from chicken carcasses in 2014 in Qingdao, China.

Methods: A total of 62 Salmonella isolates were confirmed as ESBLs by a combination disc diffusion test from 355 Salmonella isolates studied. Antimicrobial susceptibility was determined by the broth dilution method. CTX-M subtypes, Quinolone Resistance-determining Regions (QRDRs), Plasmid-mediated Quinolone Resistance (PMQR) determinants and Multi-locus Sequence Typing (MLST) were identified using PCR (Polymerase Chain Reaction) and sequencing. Plasmids were characterized by S1-nuclease PFGE, PCR-based replicon typing and transformation. Broth mating assays were carried out for all isolates to determine whether the CTX-M or PMQR marker could be transferred by conjugation.

Results: Of the 62 ESBL-producing Salmonella isolates, showing resistant to a multiple antimicrobials, 59 isolates were identified as S. indiana (ST17) followed by two S. enteritidis and one S. typhimurium. Moreover, all 62 ESBLs were subtyped to six bla ${ }_{\mathrm{CTX}-\mathrm{M}}$ types, with bla $\mathrm{CTX}_{\mathrm{C}-\mathrm{M}-123}$ being the most predominant, followed by bla ${ }_{\mathrm{CTX}-\mathrm{M}-65}$, bla $a_{\text {CTX-M-130, bla }}$ CTX-M-14, bla (T57S and S80R) and the PMQR-encoding genes [aac(6')-lb-cr,oqxA, oqxB, qnrB, and qnrS] were detected in almost all tested isolates. All isolates contained one to three large plasmids and 8 replicon types were detected with IncHI1, IncHI2, IncA/C, IncFIIAS, and IncFrep replicon types being the predominating incompatibility groups. Twenty-six isolates demonstrated the ability to transfer their CTX-M and PMQR marker at different transfer rates.

Conclusion: The emergence of CTX-M, QRDR and PMQR were found in ciprofloxacin and cefotaxime coresistant Salmonella, especially S. indiana among chicken carcasses in Qingdao. To the best of our knowledge, this is the first report of bla suggest that control strategies are necessary to limit the dissemination of these isolates through food chain.

Keywords: Salmonella; Chicken carcasses; CTX-M; QRDRs; PMQR; Horizontal gene transfer; Plasmids replicon type

\section{Introduction}

Salmonella is a bacterial genus of importance to public health causing morbidity and mortality world widely [1,2]. In Europe, this bacterium caused 91,034 human infections and 61 people death in 2012 in 27 countries [3]. In the United States, Salmonella infection is reported as the second major cause of foodborne pathogen diseases [4]. In China, more than $20 \%$ of foodborne diseases being attributed to Salmonella [5,6]. Fluoroquinolone-based compounds and third generation cephalosporins are the front line agents of treating invasive infections or severe diarrhea in young children, the elderly and those individuals with low immunity [7].
However, the widespread and discretionary usage of antimicrobial agents in medical science and veterinarian clinical medicine have increased the emergence and spread of Multidrug Resistant (MDR) Salmonella species including isolates exhibiting fluoroquinolone resistance and ESBL producing phenotypes in human as well as food animals. This development complicates the subsequent treatment of human salmonellosis $[8,9]$. Previous studies reported on the prevalence of fluoroquinolone and cephalosporins co-resistant Salmonella cultured from animals particularly chicken in China and other countries [10-12]. Moreover, these co-resistant Salmonella in chicken could not only directly infect humans, but also cause indirect infections by transmitting to humans via the food chain [8].

Resistance to cephalosporins of Salmonella and other Enterobacteriacea is mostly because of acquisition of genes encoding ESBLs locating on mobile genetic elements particularly plasmids [12]. 
Citation: $\quad$ Wang W, Xu J, Zhao L, Hu Y, Bai Y, et al. (2019) Molecular Characterization of CTX-M Producing Salmonella Isolates with Concurrent Resistance to Ciprofloxacin and Cefotaxime from Slaughtered Chicken Carcasses in Qingdao, China. J Antimicrob Agents 5: 185. doi: $10.4172 / 2472-1212.1000185$

Page 2 of 8

Over the last decade, the most frequently encountered ESBL types in Asia are the CTX-M group that are commonly carried on transmissible plasmids, which disseminate among Salmonella and other Enterobacteriacea [13]. Moreover, these CTX-M encoding plasmids may also possess resistant determinants for other antimicrobials like fluoroquinolones, which bring large challenges to clinical treatment [14]. Plasmid-mediated quinolone resistance (PMQR) can be classified into three different resistant mechanisms: $A A C\left(6^{3}\right)-I b-c r$ acetylating ciprofloxacin and norfloxacin; Qnr proteins mediating target protection and the OqxAB and QepA mediating drug efflux [15].

Although these PMQRs lead only low resistance to fluoroquinolone, their appearance (especially the qnr genes) can make bacteria survival once exposed to fluoroquinolone-based compounds and accelerate their subsequent development of high resistance phenotype to fluoroquinolone [16]. Additionally, chromosomal mutations in the Quinolone Resistance-determining Regions (QRDRs) of genes that encode gyrase or topoisomerase IV, are also frequently described in fluoroquinolone resistant Salmonella isolates [15]. It becomes especially troublesome when ESBLs and PMQR genes are present in isolates and co-transmitted through transferable plasmids.

Recently, ciprofloxacin and cefotaxime co-resistant Salmonella isolated from slaughtered chicken carcasses have been reported in several regions in China, posing a potential public health risk [17]. Here, the current study reports on the characterization of these concurrent resistant Salmonella isolates recovered from chicken carcasses in Qingdao, China.

\section{Methods}

\section{Bacterial isolates}

Sixty-two ciprofloxacin and cefotaxime co-resistant isolates were cultured from 355 Salmonella strains isolated from slaughtered chicken carcasses in 2014 in Qingdao, China. Laboratory protocols for isolating Salmonella were described previously [17]. All Salmonella isolates were confirmed through amplifying of the invA gene using PCR [18]. The serology was accomplished by the Luminex 200 system based on an xMAP ${ }^{\circ}$ Salmonella Serotyping Assay Kit (Luminex 200, Austin, USA).

Those Salmonella expressing a co-resistance phenotype were selectively enriched for study with Brain Heart Infusion Broth (BHI) (Land Bridge, Beijing, China) supplemented with $2 \mathrm{mg} / \mathrm{L}$ cefotaxime and $1 \mathrm{mg} / \mathrm{L}$ ciprofloxacin, respectively. All recovered Salmonella isolates were further screened for ESBL production by a combination disc diffusion test by cefotaxime and ceftazidime discs, with and without clavulanic acid (Land Bridge, Beijing, China) according to CLSI guidelines [19]. Escherichia coli ATCC ${ }^{\mathrm{m}} 25922$ and Klebsiella pneumoniae ATCC 700603 were applied as reference strain in Antimicrobial Susceptibility Tests (AST). All identified isolates were preserved in Brain Heart Infusion Broth (Land Bridge, Beijing, China) containing $40 \%[\mathrm{v} / \mathrm{v}]$ glycerol in -80 for subsequent study.

\section{Antimicrobial susceptibility testing}

All 62 Salmonella isolates was detected for their antimicrobial susceptibility using the broth dilution method by the Biofosun Gramnegative panels (Shanghai Biofosun Biotech, China) according to the CLSI (Clinical \& Laboratory Standards Institute) guidelines [19]. The following antimicrobials were assessed: ampicillin (AMP, 1-32 mg/L), ampicillin-sulbactam (SAM, 0.25/0.125-32/16 $\mathrm{mg} / \mathrm{L}$ ), ceftazidime
(CAZ, 0.25-32 mg/L), cefotaxime (CTX, 0.25-32 mg/L), imipenem (IPM, 0.125-16 mg/L), meropenem (MEM, 0.125-16 mg/L), trimethoprim-sulfamethoxazole (SXT, 0.125/2.38-16/304 $\mathrm{mg} / \mathrm{L}$ ), gentamicin (GEN, $0.25-32 \mathrm{mg} / \mathrm{L})$, tetracycline (TET, $0.25-32 \mathrm{mg} / \mathrm{L}$ ), ciprofloxacin (CIP, 0.03-64 mg/L), nalidixic acid (NAL, 0.25-128 $\mathrm{mg} / \mathrm{L}$ ), chloramphenicol (CHL, 0.25-32 mg/L). The Minimum Inhibitor Concentration (MIC) values for imipenem and meropenem were subsequently confirmed by Etest" (bioMérieux, France).

\section{DNA purification}

Frozen isolated were incubated for $18-24 \mathrm{~h}$ at 37 Luria-Bertani broth. A commercial bacterial DNA extraction kit (Bacterial DNA Kit D3350, Guangzhou, China) was employed to extract pure genomic DNA from the bacterial culture. A Qubit 3.0 fluorometer (Thermo Fisher Scientific, NH, USA) was used to detect the quality of DNA. DNA samples were diluted into a concentration of $50 \mathrm{mg} / \mathrm{L}$ with sterile deionized water for subsequent PCR assay.

\section{PCR and DNA sequencing}

Extracted Genomic DNA of the Salmonella isolates were used as template for the detection of ESBL bla ${ }_{\text {CTX-M }}$ gene cluster by PCR [20]. PCR amplification of QRDRs ( $g y r A$, gyr $B$, parC, and parE) and PMQR determinants $\left[q e p A, \operatorname{aac}\left(6^{\prime}\right)-I b-c r, o q x A / B\right.$, and $\left.q n r A / B / C / D / S\right]$ were performed on the ciprofloxacin-resistant strains [21-23]. All PCR products were commercially sequenced (Thermo Fisher Scientific China, Shanghai, China) and subsequently analyzed by DNAstar (DNAstar Inc., Madison, WI, USA) and then, the resulted sequences were blasted with reference sequences from NCBI.

\section{Plasmid profiling}

The plasmid profiles of all 62 strains were determined by S1nuclease (Promega, Madison, WI, USA) digestion and PFGE. Briefly, the bacterial cells were embedded in agarose plugs and digested with 8 $\mathrm{U}$ of S1 nuclease at 37 for $45 \mathrm{~min}$. Each plasmid sample was analyzed by PFGE in a Chef-Mapper XA System (Bio-Rad, USA) at 14, with a switch time between $1 \mathrm{~s}$ and $12 \mathrm{~s}$, at $6 \mathrm{~V} / \mathrm{cm}$ on a 120 angle in $0.5 \times$ TBE buffer for $18 \mathrm{~h}$. A unit length of linear plasmid was identified by each visible DNA band. Salmonella Braenderup H9812 was used to determine the approximate sizes of plasmids.

\section{PCR-based plasmid replicon typing (PBRT)}

The incompatibility groups of the studied plasmids of all Salmonella strains were identified by the PBRT according the protocols described previously [24].

\section{Conjugation-based mating experiments and verification}

All 62 isolates were studied for their capability to transfer the plasmids mediated CTX-M enzymes and PMQR markers by broth mating to a plasmid-free E. coli J53 strain $[25,26]$. PCR of CTX-M and PMRQs markers, AST, PBRT, and S1-PFGE were again performed for all the transconjugations to analyze the presence of CTX-M and PMQR genes, antimicrobial susceptibility and plasmid features, respectively. 
Citation: $\quad$ Wang W, Xu J, Zhao L, Hu Y, Bai Y, et al. (2019) Molecular Characterization of CTX-M Producing Salmonella Isolates with Concurrent Resistance to Ciprofloxacin and Cefotaxime from Slaughtered Chicken Carcasses in Qingdao, China. J Antimicrob Agents 5: $185 . \quad$ doi: $10.4172 / 2472-1212.1000185$

Page 3 of 8

\section{MLST}

MLST was performed following the protocols described at the MLST website (http://mlst.ucc.ie/mlst/dbs/Senterica/documents/ primersEnterica_html).

Seven conserved housekeeping genes (aroC, dnaN, hemD, hisD, purE, $\operatorname{suc} A$, and $\operatorname{thr} A$ ) of Salmonella enterica were amplified and sequenced at Thermo Fisher Scientific (China) Co. Ltd (Shanghai, China). Sequences were submitted to the Salmonella MLST database website (http://mlst.warwick.ac.uk/mlst/dbs/Senterica) to assign the Sequence Types (STs).

\section{Results}

\section{Antimicrobial susceptibility testing}

In this study, all 62 Salmonella isolates $(17.5 \%, 62 / 355)$ were confirmed to be ESBL-positive and co-resistant to ampicillin, ampicillin-sulbactam, cefotaxime, ciprofloxacin, nalidixic acid, gentamicin, and chloramphenicol. Moreover, resistance to trimethoprim/sulfamethoxazole $(61 / 62,98.4 \%)$ was common, followed by ceftazidime $(48 / 62,77.4 \%)$, tetracycline $(18 / 62,29.0 \%)$. Notably, one isolate $(1 / 62,1.6 \%)$ was found to be resistant to carbapenems (imipenem and meropenem).

All Salmonella isolates expressed a MDR phenotype. The 62 Salmonella isolates were subtyped into three serotypes including 59 $(95.2 \%, 59 / 62) S$. indiana, $2(3.2 \%, 2 / 62) S$. enteritidis and $1(1.6 \%$, 1/62) $S$. typhimurium isolates. The antimicrobial resistance profiles of the collected Salmonella isolates were showed in Table 1, Table S1 and Figure 1 .

\begin{tabular}{|l|l|l|l|l|l|}
\hline Antibiotic class & Antimicrobial agent & Total, $\mathbf{n}(\mathbf{r a t e})$ & S. indiana, $\mathbf{n}(\mathbf{r a t e})$ & S. enteritidis, $\mathbf{n}$ & S. typhimurium, $\mathbf{n}$ \\
\hline Penicillin & Ampicillin & $62(100)$ & $59(100)$ & 2 & 1 \\
\hline \multirow{2}{*}{ Cephalosporin } & Cefotaxime & $62(100)$ & $59(100)$ & 2 & 1 \\
\cline { 2 - 6 } & Ceftazidime & $48(77.4)$ & $46(78.0)$ & 1 & 1 \\
\hline \multirow{2}{*}{ Fluoroquinolone } & Ciprofloxacin & $62(100)$ & $59(100)$ & 2 & 1 \\
\cline { 2 - 6 } & Nalidixic Acid & $62(100)$ & $59(100)$ & 2 & 1 \\
\hline B-lactam & Ampicillin-Sulbactam & $62(100)$ & $59(100)$ & 2 & 1 \\
\hline Aminoglicosides & Gentamicin & $62(100)$ & $59(100)$ & 2 & 1 \\
\hline Phenicol & Chloramphenicol & $62(100)$ & $59(100)$ & 2 & 1 \\
\hline Folate pathway inhibitors & Trimethoprim/Sulfamethoxazole & $61(98.4)$ & $59(100)$ & 1 & 1 \\
\hline Tetracycline & Tetracycline & $18(29.0)$ & $15(25.4)$ & 2 & 1 \\
\hline \multirow{2}{*}{ Carbapenems } & Imipenem & $1(1.6)$ & $1(1.7)$ & $1(1.7)$ & 0 \\
\cline { 2 - 6 } & Meropenem & $1(1.6)$ & & 0 \\
\hline
\end{tabular}

Table 1: Antimicrobial resistance of 62 Salmonella isolates within serogroups.

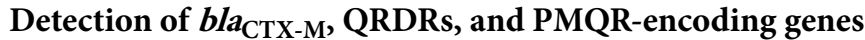

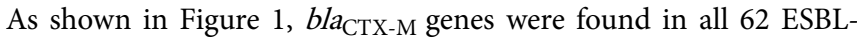
producing Salmonella isolates by PCR. Sequence analysis revealed six bla $_{\text {CTX-M }}$ subtypes including bla $a_{\text {CTX-M-123 }}(\mathrm{n}=43)$, bla $_{\text {CTX-M-65 }}(\mathrm{n}=38)$, bla $_{\text {CTX-M-130 }}(\mathrm{n}=10)$, bla $_{\text {CTX-M-14 }}(\mathrm{n}=7), \operatorname{bla}_{\mathrm{CTX}-\mathrm{M}-24}(\mathrm{n}=5)$, and bla $a_{\mathrm{CTX}}$ $\mathrm{M}-79(\mathrm{n}=1)$.

Moreover, four QRDRs point mutations, two in gyrA (giving rise to $\mathrm{S} 83 \mathrm{~F}$ and $\mathrm{D} 87 \mathrm{~N}$ amino acid substitutions) and two in parC (giving rise to T57S and S80R amino acid substitutions), respectively, were found in almost all 62 Salmonella isolates with two exceptions wherein the latter ParC mutations (T57S and S80R) were absent in isolates $\mathrm{CH} 60$, while GyrA mutations (S83F) and ParC mutations (T57S and S80R) were absent in isolates $\mathrm{CH} 62$.

Additionally, all 62 Salmonella isolates were detected for the presence and frequency distribution of each $\mathrm{PMQR}$-encoding gene. No $q e p A$, $q n r A, q n r C$, and $q n r D$ genes were detected in all the isolates.
While the $\operatorname{aac}\left(\sigma^{\prime}\right)-I b-c r, o q x A, o q x B, q n r B$, and $q n r S$ were found in almost all tested isolates except that the qnrB gene was not identified in two isolates (denominated as isolate $\mathrm{CH} 36$ and $\mathrm{CH} 50$, respectively).

\section{Plasmids profiles and PCR-based plasmid replicon types}

All 62 Salmonella isolates contained detectable large plasmids by S1 nuclease-based plasmid analysis (Figure 1). In brief, 40 isolates $(64.5 \%$, $40 / 62)$ possessed one plasmid; 19 isolates $(30.6 \%, 19 / 62)$ possessed two plasmids and 3 isolates $(4.8 \%, 3 / 62)$ possessed three plasmids, respectively.

Plasmid replicon types of all 62 isolates were detected by PCR to evaluate the heterogeneity among the profiles (Figure 1 ). These results showed that 8 of 18 replicons were detected, while the IncX, IncL/M, IncN, IncW, IncY, IncP, IncFIC, IncT, IncK/B, and IncB/O types were not detected in this study. 
Citation: $\quad$ Wang W, Xu J, Zhao L, Hu Y, Bai Y, et al. (2019) Molecular Characterization of CTX-M Producing Salmonella Isolates with Concurrent Resistance to Ciprofloxacin and Cefotaxime from Slaughtered Chicken Carcasses in Qingdao, China. J Antimicrob Agents 5: $185 . \quad$ doi: $10.4172 / 2472-1212.1000185$

Page 4 of 8

IncHI1 $(n=34)$, IncHI2 $(n=23)$, IncA/C $(n=18)$, IncFIIAS $(n=16)$, Moreover, 43 of 62 isolates were found to have for more than 1 and IncFrep $(\mathrm{n}=12)$ replicon types were the frequently present types. replicon type.

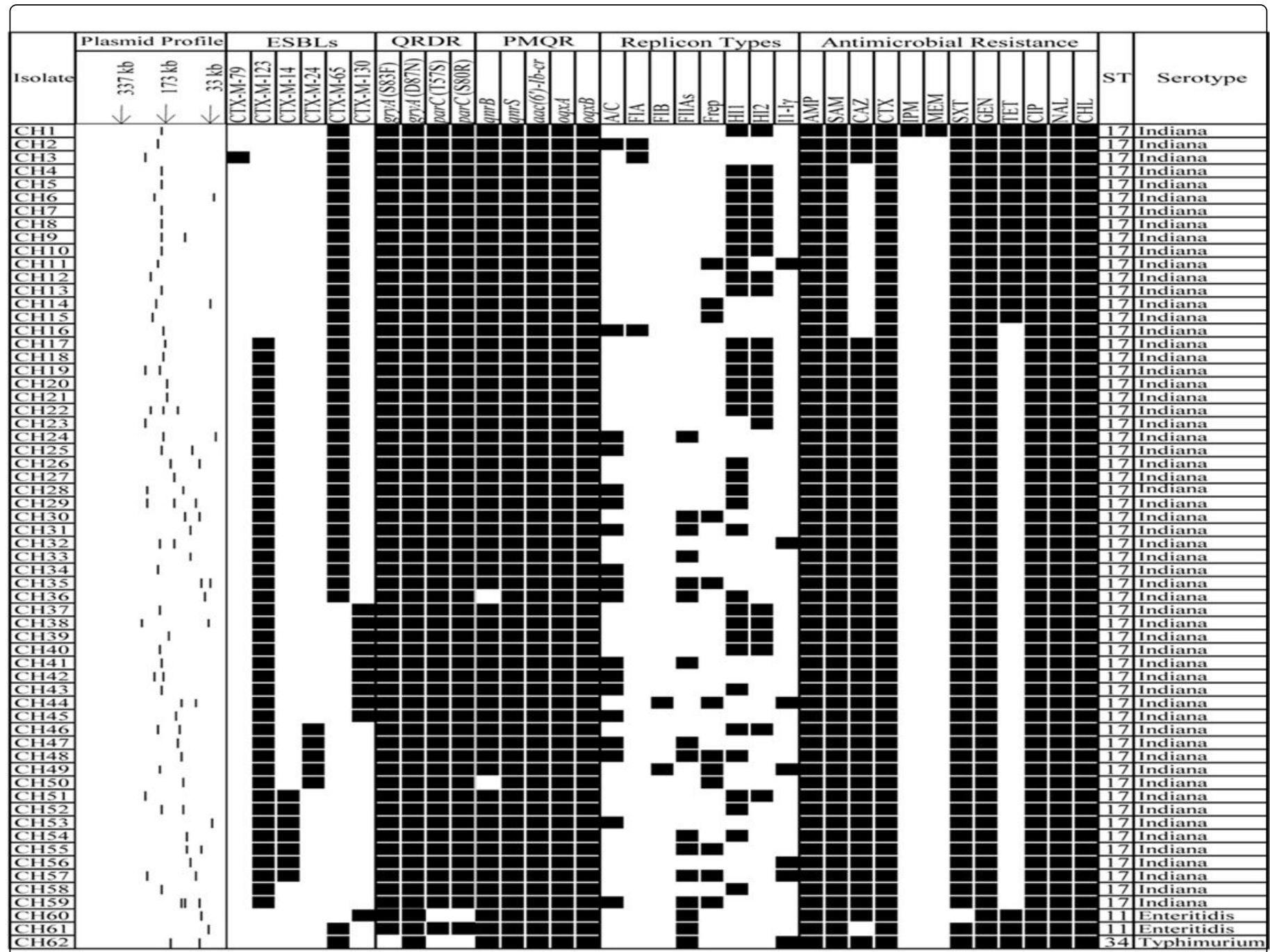

Figure 1. A heat-map summary of the schematic showing the S1 nuclease plasmid profile; ESBL profiles, QRDR mutations and PMQR genes identified by PCR and DNA sequencing, corresponding Inc plasmid type(s), antimicrobial resistance profile, MLST and serotypes for all 62 Salmonella isolated from chicken carcasses in Qingdao, China. Black squares shown indicate the features present in the ESBL marker, the QRDR mutations and PMQR genes, the Inc type and the corresponding antimicrobial resistance profile. White squares denote features that are lacking in the corresponding bacterial isolate. Antimicrobial compounds used are abbreviated as follows: AMP: Ampicillin; SAM: Ampicillin-sulbactam; CAZ: Ceftazidime; CTX: Cefotaxime; IPM: Imipenem; MEM: Meropenem; SXT: Trimethoprim/sulfamethoxazole; GEN: Gentamicin; TET: Tetracycline; CIP: Ciprofloxacin; NAL: Nalidixic acid; CHL: Chloramphenicol.

Conjugational transfer of plasmid encoding CTX-M and PMQRs genes carried by the Salmonella isolates

Of 62 Salmonella isolates tested for conjugation assay, 26 isolates were found that could transfer the cefotaxime and quinolone (ciprofloxacin/nalidixic acid) resistance determinants to E. coli J53 with the conjugation frequencies ranging from $4.4 \times 10^{-8}$ to $7.5 \times 10^{-2}$ (Figure 2).

Subsequently, the AST results of all transconjugants showed that resistance to antimicrobial agents including ampicillin, trimethoprim- sulfamethoxazole, gentamicin, tetracycline, and chloramphenicol in addition to cephalosporins and fluoroquinolone, were also transferred to the E. coli J53 strain.

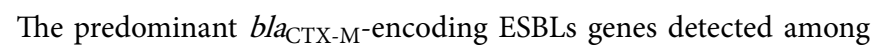
the transconjugants were bla ${ }_{\mathrm{CTX}-\mathrm{M}-65}(18 / 26)$ and bla $_{\mathrm{CTX}-\mathrm{M}-123}(16 / 26)$. Following the CTX-M genes, the PMQR genes were also detected among the transconjugants, showing in Figure 2. 
Citation: Wang W, Xu J, Zhao L, Hu Y, Bai Y, et al. (2019) Molecular Characterization of CTX-M Producing Salmonella Isolates with Concurrent Resistance to Ciprofloxacin and Cefotaxime from Slaughtered Chicken Carcasses in Qingdao, China. J Antimicrob Agents 5: $185 . \quad$ doi: $10.4172 / 2472-1212.1000185$

Page 5 of 8

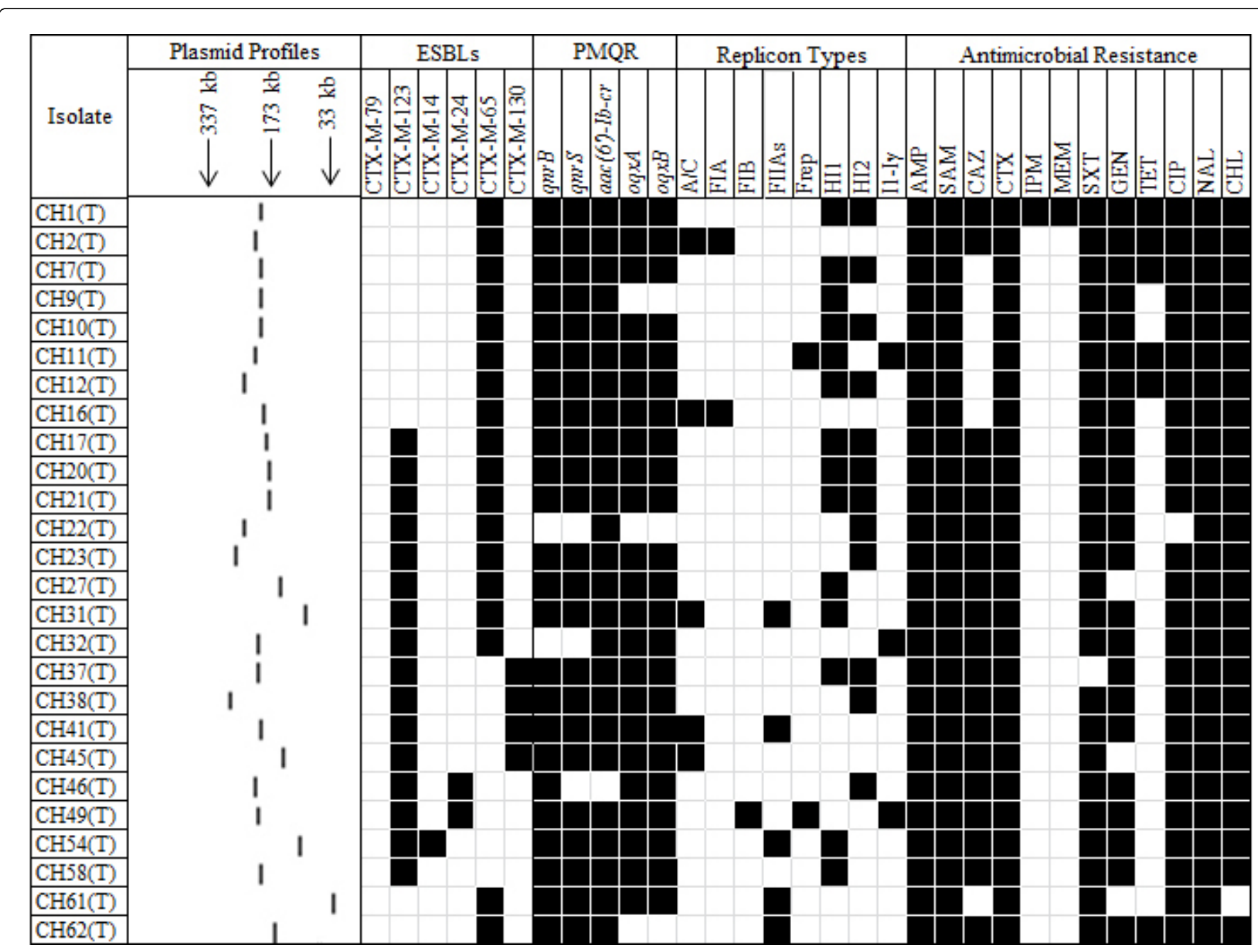

Figure 2: A heat-map showing the resultant transconjugants, characterized on the basis of their plasmid profiles; ESBL-markers and PMQR genes identified by PCR and DNA sequencing; antimicrobial resistance profile; and plasmid replicon type(s). Antimicrobial compounds are abbreviated as described in the legend to Figure 1.

\section{MLST}

All 62 Salmonella isolates were subtyped by MLST and seven STs were identified (Figure 1). The genotyping results showed that all $59 S$. indiana isolates were identified as ST17; both $2 S$. enteritidis were identified as ST11; and $1 S$. typhimurium isolate was identified as ST34, respectively.

\section{Discussion}

The emergence of CTX-M-producing Salmonella has been increasingly reported among bacteria cultured from poultry throughout the world including China $[10,15,16,27]$. Of note, poultry especially chicken and chicken products are often involved in human salmonellosis, thereby presenting an important challenge for the clinical and veterinary communities [28-30]. Consequently, the clonal dissemination of CTX-M producing and fluoroquinolone-resistant Salmonella via chicken can give rise to serious infections whilst reducing the treatment options available for clinicians. Moreover, ciprofloxacin and cefotaxime co-resistant Salmonella isolates could be transferred to non-contaminated chicken carcasses via crosscontamination during the slaughtering process, thus increasing the spread of these isolates in the environment and further along the food chain [31]. Previous reports have described the nature of these coresistant resistant Salmonella in farm or retail chicken [11,15,32,33]. However, there is limited reports describing the nature of CTX-M producing-Salmonella isolates co-resistant to fluoroquinolones from slaughtered chicken carcasses in China $[10,16]$. In this study, we described the antimicrobial susceptibility and associated molecular mechanisms for a collection of Salmonella isolates that were resistant to both ciprofloxacin and cefotaxime from chicken carcasses in a slaughterhouse in Qingdao, China.

In this study, an extensive resistant phenotype to a multi antimicrobial compounds observed and which was likely linked to the usage of these agents in the poultry production chain in China $[4,15,34]$. Meanwhile, a total of $17.5 \%(62 / 355)$ ciprofloxacin and cefotaxime co-resistant Salmonella isolates were found to be ESBLpositive, a figure that was similar to previous reports in China $[16,17,35]$. The high frequency of fluoroquinolone resistance in 
Citation: $\quad$ Wang W, Xu J, Zhao L, Hu Y, Bai Y, et al. (2019) Molecular Characterization of CTX-M Producing Salmonella Isolates with Concurrent Resistance to Ciprofloxacin and Cefotaxime from Slaughtered Chicken Carcasses in Qingdao, China. J Antimicrob Agents 5: 185. doi: $10.4172 / 2472-1212.1000185$

Page 6 of 8

Salmonella has been considered as an important feature in China and several other developing countries that do not strictly control the application of antimicrobial agents in veterinary settings and hospitals $[36,37]$. In contrast, more than $3 \%$ of Salmonella isolates were identified as intermediate or resistant to ciprofloxacin in the United States, where the usage of this class of antimicrobial is well controlled [38]. Therefore, it is necessary to enforce controls on the use of antimicrobial compounds of critical importance the poultry industry. Additionally, in this study, more than $95 \%$ of the Salmonella isolates were identified as serovar Indiana, which were subtyped as ST17 subsequently. This ST17 clone has been widely reported in foodproducing animals and human cases in China [17,29,34]. Moreover, the contribution of chicken-originated $S$. indiana strains in the community infections has been established [16]. Interestingly, one $S$. indiana isolate in our study collection was found to have the resistant phenotype to all tested antimicrobial agents including imipenem and meropenem. The latter phenotype was identified as a New Delhi Metallo-beta-lactamase (NDM) producing isolate expressing an Extensively-drug Resistance (XDR) phenotype [39]. Therefore, the spread of MDR $S$. indiana strains becomes a potential threat to chicken health and would consequently represent an important public health hazard via the food chain.

CTX-M has replaced the TEM (Temoneira) and SHV (Sulfhydryl Variable) as the predominant ESBLs since they were discovered in the 1980s [40,41]. ESBL screening and characterization of isolates in the current study showed that different CTX-M subtypes were identified. Previous studies reported that the $b_{\text {CTX-M- } 65}$ and $b l a_{\text {CTX-M-14 }}$ were the most frequently detected genotypes among Salmonella in chicken in China $[16,29,42]$. However, in the current study, of the 62 CTX-M producing isolates, bla genotype, followed by bla $a_{\mathrm{CTX}-\mathrm{M}-65}$, and bla $a_{\mathrm{CTX}-\mathrm{M}-130}$, suggesting that the CTX-M subtypes may have particular epidemic characteristics in different geographical regions. Moreover, both of the bla $a_{\mathrm{CTX}-\mathrm{M}-123}$ and

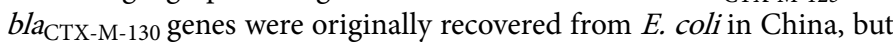
rarely detected in Salmonella [43-45]. As far as we know, this is the first study to identify bla $a_{\text {CTX-M-123 }}$ and bla Additionally, other CTX-M subtypes such as bla ${ }_{\text {CTX-M-14, }}$ bla ${ }_{\text {CTX-M-24 }}$ and bla $a_{\text {CTX-M-79 }}$ were also found in this study and these have been previously detected in Salmonella or E. coli from both food-producing animal and human in China [16,42]. Salmonella isolates in this study

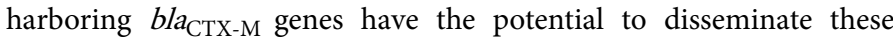
markers amongst different Gram-negative bacteria, a feature that confounds our ability to control the spread of these resistance genotypes.

The fluoroquinolone resistant determinants were also analyzed among 62 ESBLs in this study. Amino acid substitutions in GyrA (S83F and $\mathrm{D} 87 \mathrm{~N}$ ) and $\operatorname{ParC}$ (T57S and S80R) were found in almost all tested isolates, and which were detected previously $[16,26,46]$. In this study, the PMQR-encoding genes were detected in all 62 isolates including $a a c\left(\sigma^{\prime}\right)-I b-c r, o q x A / B, q n r B / S$. The oqx $A / B$ and $a a c\left(\sigma^{\prime}\right)-I b-c r$ genes were commonly detected in previous studies $[16,35,47]$. Accordingly, $O q_{x} A B$ encoded by the oqxA and oqxB genes is the first identified mechanism of resistance to olaquindox $[48,49]$. Olaquindox is used in animal to promote their growth for decades in China, suggesting that a constant selective pressure may contribute the acquisition and dissemination of $o q x A / B[29,50]$. The $o q x A / B$ and $a a c\left(\sigma^{\prime}\right)-I b-c r$ genes are often located on plasmids, which may contain other resistance markers such as ESBL-encoding genes, as well as chloramphenicol and biocides mechanisms [29,49,51]. Moreover, the $q n r B / S$ genes were found in almost all isolates in this study. These two qnr genes together with $a a c\left(6^{\prime}\right)-I b-c r$ and $o q x A / B$, co-existing in a single isolate was seldom reported in Salmonella from the USA, Europe or Japan [52,53]. Additionally, several studies indicated that PMQR-encoding genes could only confer only low-level prevalence of fluoroquinolone resistance $[16,54]$. Nonetheless, they could facilitate the acceleration of the emergence of high fluoroquinolone resistance by subsequent mutations in the topoisomerase genes [52]. Further studies showed that mutations only in $G y r A$ confer low fluoroquinolone resistance while additional GyrA mutation(s) together with $\operatorname{ParC}$ could increase their resistance to a high level [53-56]. Interestingly, we found two isolates with mutations only in GyrA in this study, and which expressed a lower-level resistance to ciprofloxacin $(\mathrm{MIC}=2 \mathrm{mg} / \mathrm{L})$ than those with multiple mutations in both GyrA and $\operatorname{ParC}(\mathrm{MIC}>64$ $\mathrm{mg} / \mathrm{L})$.

Characterization of high molecular-weight plasmids by S1-PFGE

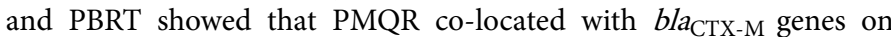
plasmids of IncHI1, IncHI2, IncA/C, IncFIIAS, IncFrep replicon types of different sizes. Furthermore, several of the PMQR and bla CTX-M genes $(26 / 62)$ could be horizontally transferred. Accordingly, the IncHI1, IncHI2 and IncA/C plasmids have been intensively studied due to their temperature-dependent conjugation, which are also associated with ESBLs and MDR determinants emerging in animals and humans and which were reported to have originated in Salmonella in China and elsewhere [57-59]. Besides, IncFIIAS and IncFrep plasmids were rarely reported in Salmonella but commonly present in $E$. coli in China, suggesting that these plasmids might have been acquired by Salmonella from E. coli more recently [60]. Hence, these conjugative plasmids of different replicons types have the potential to drive such increased transfer of PMQR and bla $a_{C T X-M}$ genes within and/or between bacterial species colonizing chicken, posing a risk of the transfer of these resistant isolates into the food chain and leading to human infections.

\section{Conclusions}

The CTX-M producing MDR $S$. indiana isolates were predominant among chicken carcasses in this study. As far as we know, this study firstly reported bla $a_{\mathrm{CTX}-\mathrm{M}-123}$ and bla $a_{\mathrm{CTX}-\mathrm{M}-130}$ in Salmonella. A high prevalence of PMQR genes was found among the studied isolates, while the data confirms the observation that mutations present in both of GyrA and ParC are essential for high resistance level of ciprofloxacin. The conjugative resistant plasmids harboring bla $a_{\mathrm{CTX}-\mathrm{M}}$ and PMQR genes were responsible for the dissemination of Salmonella isolates, posing a potential threat for public health. Our data suggest that there should be a reassessment and reduction usage of cephalosporins, fluoroquinolones, and other antimicrobial compounds, critical to human health, in the poultry industry in China, as an important step to improve food safety controls.

\section{Funding}

This study was funded by the National Key R\&D Program of China (2016YFD0401102), and China Food Safety Talent Competency Development Initiative: CFSA 523 Program.

\section{Author Contributions}

WW, XJ, L-FQ, and SF designed the experiments and wrote the manuscript. ZL, H-YJ, BY, GX, and D-YP carried out the experiments. WW, P-ZX, Y-SF and L-MH analyzed the experimental results. 
Citation: $\quad$ Wang W, Xu J, Zhao L, Hu Y, Bai Y, et al. (2019) Molecular Characterization of CTX-M Producing Salmonella Isolates with Concurrent Resistance to Ciprofloxacin and Cefotaxime from Slaughtered Chicken Carcasses in Qingdao, China. J Antimicrob Agents 5: 185. doi: $10.4172 / 2472-1212.1000185$

Page 7 of 8

\section{References}

1. Chon JW, Jung HI, Kuk M, Kim YJ, Seo KH, et al. (2015) High occurrence of extended-spectrum $\beta$-lactamase-producing Salmonella in broiler carcasses from poultry slaughterhouses in South Korea. Foodborne Pathog Dis 12: 190-196.

2. Chiu LH, Chiu CH, Horn YM, Chiou CH, Lee CY, et al. (2010) Characterization of 13 multi-drug resistant Salmonella serovars from different broiler chickens associated with those of human isolates. BMC Microbiol 10: 86.

3. European Food Safety Authority (2014) The European Union summary report on trends and sources of zoonoses, zoonotic agents and foodborne outbreaks in 2012. EFSA J 12: 3547.

4. Wang Y, Cao C, Alali WQ, Cui S, Li F, et al. (2017) Distribution and antimicrobial susceptibility of foodborne Salmonella serovars in eight provinces in China from 2007 to 2012 (Except 2009). Foodborne Pathog Dis 14: 393-399.

5. Zhu M, Cui S, Lin L, Xu B, Zhao J, et al. (2013) Analysis of the aetiology of diarrhea in outpatients in 2007, Henan province, China. Epidemiol Infect 141: 540-548.

6. Wang SJ, Duan HL, Zhang W, Li JW (2007) Analysis of bacterial foodborne disease outbreaks in China between 1994 and 2005. FEMS Immunol Med Microbiol 51: 8-13.

7. Clemente L, Manageiro V, Ferreira E, Jones-Dias D, Correia I, et al. (2013) Occurrence of extended-spectrum beta-lactamases among isolates of Salmonella enterica subsp. enterica from food-producing animals and food products, in Portugal. Int J Food Microbiol 167: 221-228.

8. Seiffert SN, Hilty M, Perreten V, Endimiani A (2013) Extended-spectrum cephalosporin-resistant gram-negative organisms in livestock: An emerging problem for human health? Drug Resist Update 16: 22-45.

9. Sarkar A, Pazhani GP, Chowdhury G, Ghosh A, Ramamurthy T (2015) Attributes of carbapenemase encoding conjugative plasmid pNDM-SAL from an extensively drug-resistant Salmonella enterica Serovar Senftenberg. Front Microbiol 6: 969.

10. Bai L, Lan R, Zhang X, Cui S, Xu J, et al. (2015) Prevalence of Salmonella Isolates from Chicken and Pig Slaughterhouses and Emergence of Ciprofloxacin and Cefotaxime Co-Resistant S. enterica Serovar Indiana in Henan, China. PLoS ONE 10: e0144532.

11. Kuang X, Hao H, Dai M, Wang Y, Ahmad I, et al. (2015) Serotypes and antimicrobial susceptibility of Salmonella spp. isolated from farm animals in China. Front Microbiol 6: 602.

12. Noda T, Murakami K, Etoh Y, Okamoto F, Yatsuyanagi J, et al. (2015) Increase in resistance to extended-spectrum cephalosporins in salmonella isolated from retail chicken products in Japan. PLoS ONE 10: e0116927.

13. Cantón R, González-Alba JM, Galán JC (2012) CTX-M enzymes: origin and diffusion. Front Microbiol 3: 1-19.

14. Harrois D, Breurec S, Seck A, Delauné A, Le Hello S, et al. Prevalence and characterization of extended-spectrum beta-lactamase-producing clinical Salmonella enterica isolates in Dakar. Senegal, from 1999 to 2009. Clin Microbiol Infect 20: O109-O116.

15. Zhang WH, Lin XY, Xu L, Delauné A, Le Hello S, et al. (2014) CTX-M-27 producing Salmonella enterica serotypes typhimurium and indiana are prevalent among food-producing animals in China. Front Microbiol 7: 436.

16. Bai L, Zhao J, Gan X, Wang J, Zhang X, et al. (2016) Emergence and diversity of Salmonella enterica serovar Indiana isolates with concurrent resistance to ciprofloxacin and cefotaxime from patients and foodproducing animals in China. Antimicrob Agents Chemother 60: 3365-3371.

17. Wang Y, Chen Q, Cui S, Xu X, Zhu J, et al. (2014) Enumeration and characterization of Salmonella isolates from retail chicken carcasses in Beijing, China. Foodborne Pathog Dis 11: 126-132.

18. Malorny B, Hoorfar J, Bunge C, Helmuth R (2003) Multicenter validation of the analytical accuracy of Salmonella PCR: towards an international standard. Appl Environ Microbiol 69: 290-296.
19. Clinical and Laboratory Standards Institute (2015) Performance standards for antimicrobial susceptibility testing: Twenty-second informational supplement M100-S22. CLSI, Wayne, PA, USA.

20. Xu L, Ensor V, Gossain S, Nye K, Hawkey P (2005) Rapid and simple detection of blaCTX-M genes by multiplex PCR assay. J Med Microbiol 54: 1183-1187.

21. Giraud E, Brisabois A, Martel JL, Chaslus-Dancla E (1999) Comparative studies of mutations in animal isolates and experimental in vitro- and in vivo-selected mutants of Salmonella spp. suggest a counterselection of highly fluoroquinolone-resistant strains in the field. Antimicrob Agents Chemother 43: 2131-2137.

22. Park CH, Robicsek A, Jacoby GA, Sahm D, Hooper DC (2006) Prevalence in the United States of aac(6')-Ib-cr encoding a ciprofloxacin-modifying enzyme. Antimicrob Agents Chemother 50: 3953-3955.

23. Cui S, Li J, Sun Z, Hu C, Jin S, et al. (2008) Ciprofloxacin-resistant Salmonella enterica serotype Typhimurium. China. Emerg Infect Dis 14: 493-495.

24. Carattoli A, Bertini A, Villa L, Falbo V, Hopkins KL, et al. (2005) Identification of plasmids by PCR-based replicon typing. J Microbiol Methods 63: 219-228.

25. Barton BM, Harding GP, Zuccarelli AJ (1995) A general method for detecting and sizing large plasmids. Anal Biochem 226: 235-240.

26. Wang J, Stephan R, Karczmarczyk M, Yan Q, Hächler H, et al. (2013) Molecular characterization of blaESBL-harboring conjugative plasmids identified in multi-drug resistant Escherichia coli isolated from foodproducing animals and healthy humans. Front Microbiol 4: 188.

27. Doublet B, Praud K, Nguyen-Ho-Bao T, Argudín MA, Bertrand S, et al. (2014) Extended-spectrum $\beta$-lactamase- and AmpC $\beta$-lactamaseproducing D-tartrate-positive Salmonella enterica serovar Paratyphi B from broilers and human patients in Belgium, 2008-10. J Antimicrob Chemother 69:1257-64.

28. Beutlich J, Jahn S, Malorny B, Hauser E, Hühn S, et al. (2011) Antimicrobial resistance and virulence determinants in European Salmonella genomic island 1-positive Salmonella enterica isolates from different origins. Appl Environ Microbiol 77: 5655-5664.

29. Jiang HX, Song L, Liu J, Zhang XH, Ren YN et al. (2014) Multiple transmissible genes encoding fluoroquinolone and third-generation cephalosporin resistance co-located in non-typhoidal Salmonella isolated from food-producing animals in China. Int J Antimicrob Agents 43: 242-247.

30. M'ikanatha NM, Sandt CH, Localio AR, Tewari D, Rankin SC, et al. (2010)Multidrug-resistant Salmonella isolates from retail chicken meat compared with human clinical isolates. Foodborne Patho Dis 7: 929-934.

31. Hue O, Le Bouquin S, Lalande F, Allain V (2012) Prevalence of Salmonella spp. on broiler chicken carcasses and risk factors at the slaughterhouse in France in 2008. Food Control 22: 1158-1164.

32. Wu H, Wang Y, Wu Y, Qiao J, Li H, et al. (2015) Emergence of $\beta$ lactamases and extended-spectrum $\beta$-lactamases (ESBLs) producing Salmonella in retail raw chicken in China. Foodborne Pathog Dis 12: 228-34.

33. Qiao J, Zhang Q, Alali WQ, Wang J, Meng L, et al. (2017) Characterization of extended-spectrum $\beta$-lactamases (ESBLs)-producing Salmonella in retail raw chicken carcasses. Int J Food Microbiol 248: 72-81.

34. Zhang WH, Zhang CZ, Liu ZJ, Gu XX, Li W, et al. (2017) In Vitro development of ciprofloxacin resistance of Salmonella enterica serovars Typhimurium, Enteritidis, and Indiana isolates from food animals. Microb Drug Resist 23: 687-694.

35. Li L, Liao XP, Liu ZZ, Huang T, Li X, et al. (2014) Co-spread of oqxAB and blaCTX-M-9G in non-Typhi Salmonella enterica isolates mediated by ST2-IncHI2 plasmids. Int J Antimicrob Agents 44: 263-8.

36. Cui S, Li J, Sun Z, Jin S, Li F, et al. (2009) Characterization of Salmonella enterica isolates from infants and toddlers in Wuhan, China. J Antimicrob Chemother 63: 87-94. 
Citation: $\quad$ Wang W, Xu J, Zhao L, Hu Y, Bai Y, et al. (2019) Molecular Characterization of CTX-M Producing Salmonella Isolates with Concurrent Resistance to Ciprofloxacin and Cefotaxime from Slaughtered Chicken Carcasses in Qingdao, China. J Antimicrob Agents 4: 185. doi: $10.4172 / 2472-1212.1000185$

Page 8 of 8

37. Von Seidlein L, Kim DR, Ali M, Lee H, Wang X, et al. (2006) A multicentre study of Shigella diarrhoea in six Asian countries: Disease burden, clinical manifestations, and microbiology. PLoS Med 3: e353.

38. Medalla F, Hoekstra RM, Whichard JM, Barzilay EJ, Chiller TM, et al. (2013) Increase in resistance to ceftriaxone and nonsusceptibility to ciprofloxacin and decrease in multidrug resistance among Salmonella strains, United States, 1996-2009. Foodborne Pathog Dis 10: 302-9.

39. Wang W, Baloch Z, Peng ZX, Yujie Hu, Jin Xu, et al. (2017) Genomic characterization of a large plasmid containing a blaNDM-1 gene carried on Salmonella enterica serovar Indiana C629 isolate from China. BMC Infect Dis 17: 479.

40. Martínez-Martínez L, Pascual A, Jacoby GA (1998) Quinolone resistance from a transferable plasmid. Lancet 351: 797-799.

41. D’Andrea MM, Arena F, Pallecchi L, Rossolini GM (2013) CTX-M-type b-lactamases: a successful story of antibiotic resistance. Int J Med Microbiol 303: 305-317.

42. Wang Y, Zhang A, Yang Y, Lei C, Jiang W, et al. (2017) Emergence of Salmonella enterica serovar Indiana and California isolates with concurrent resistance to cefotaxime, amikacin and ciprofloxacin from chickens in China. Int J Food Microbiol 262: 23-30.

43. He D, Partridge SR, Shen J, Zhenling Z, Lanping L, et al. (2013) CTXM-123, a novel hybrid of the CTX-M-1 and CTX-M-9 Group $\beta$ lactamases recovered from Escherichia coli isolates in China. Antimicrob Agents Chemother 57: 4068-71.

44. Sun J, Fang LX, Wu Z, Yang RS, Deng H, et al. (2017) Genetic analysis of the Incx4 plasmids: implications for a unique pattern in the mcr-1 acquisition. Sci Rep 7: 424.

45. Wang J, Li Y, Xu X, Liang B, Wu F, et al. (2017) Antimicrobial resistance of Salmonella enterica serovar Typhimurium in Shanghai, China. Front Microbiol 8: 510.

46. Kozoderovic G, Velhner M, Jelesic Z, Golić N, Lozo J, et al. (2012) Prevalence of quinolone resistance and mutations in the topoisomerase genes in Salmonella enterica serotype Enteritidis isolates from Serbia. Int J Antimicrob Agents 40: 455-457.

47. Strahilevitz J, Jacoby GA, Hooper DC, Robicsek A (2009)Plasmidmediated quinolone resistance: a multifaceted threat. Clin Microbiol Rev 22: 664-89.

48. Hansen LH, Sorensen SJ, Jorgensen HS, Jensen LB (2005) The prevalence of the OqxAB multidrug efflux pump amongst olaquindox-resistant Escherichia coli in pigs. Microb Drug Resist 11: 378-82.

49. Hansen LH, Jensen LB, Sorensen HI, Sørensen SJ (2007)Substrate specificity of the OqxAB multidrug resistance pump in Escherichia coli and selected enteric bacteria. J Antimicrob Chemother 60: 145-7.
50. Wong MH, Yan M, Chan EW, Biao K, Chen S (2014) Emergence of clinical Salmonella enterica serovar Typhimurium isolates with concurrent resistance to ciprofloxacin, ceftriaxone, and azithromycin. Antimicrobial Agents Chemother 58: 3752-3756.

51. Crofts TS, Gasparrini AJ, Dantas G (2017) Next-generation approaches to understand and combat the antibiotic resistome. Nat. Rev. Microbiol 15: 422-434.

52. Veldman K, Cavaco LM, Mevius D, Battisti A, Franco A, et al. (2011) International collaborative study on the occurrence of plasmid-mediated quinolone resistance in Salmonella enterica and Escherichia coli isolated from animals, humans, food and the environment in 13 European countries. J Antimicrob Chemother 66: 1278-86.

53. Asai T, Sato C, Masani K, Usui M, Ozawa M, et al. (2010) Epidemiology of plasmid-mediated quinolone resistance in Salmonella enterica serovar Typhimurium isolates from food-producing animals in Japan. Gut Pathog 2: 17 .

54. Hernández A, Sánchez MB, Martínez JL (2011) Quinolone resistance: much more than predicted. Front Microbiol 2: 22.

55. Liu F, Li C, Liu L, Li Rui, Luo PZ, et al. (2011) Fluoroquinolone resistance and topoisomerase IV mutations of Salmonella isolated from chickens. J Northeast Agric Univ 42: 94-97.

56. Yang B, Xi M, Cui S, Xiuli Z (2012) Mutations in gyrase and topoisomerase genes associated with fluoroquinolone resistance in Salmonella serovars from retail meats. Food Res Int 45: 935-939.

57. Kubasova T, Cejkova D, Matiasovicova J, Sekelova Z, Polansky O, et al. (2016) Antibiotic resistance, core-genome and protein expression in IncHI1 plasmids in Salmonella Typhimurium. Genome Biol Evol 8: 1661-1671.

58. Han J, Pendleton SJ, Deck J, Singh R, Gilbert J, et al. (2018) Impact of cocarriage of IncA/C plasmids with additional plasmids on the transfer of antimicrobial resistance in Salmonella enterica isolates. Int J Food Microbiol 271: 77-84.

59. Chen W, Fang T, Zhou X, Daofeng Z, Xianming S, et al. (2016) IncHI2 plasmids are predominant in antibiotic-resistant Salmonella isolates. Front Microbiol 7: 1566.

60. Bai L, Wang L, Yang X, Wang J, Gan X, et al. (2017) Prevalence and molecular characteristics of extended-spectrum $\beta$-lactamase genes in Escherichia coli isolated from diarrheic patients in China. Front Microbiol 8: 144. 